\title{
THE MEASUREMENT OF GROWTH ON FOREST AREAS BY MEANS OF RECURRENT LINE PLOT SURVEYS
}

\author{
By A. BickerstafF \\ Dominion Forest Service, Ottawa, Canada.
}

\section{INTRODUCTION}

$\mathrm{T}$ HE remeasurement of sample forest areas is the most accurate method of determining periodic growth, but unless certain fundamental features are kept in mind the validity of the growth figures and the subsequent deductions from them may be seriously impaired. The purpose of this article is to outline some of the difficulties which arise in such work and to suggest, where possible, procedures which will minimize the errors concerned.

\section{General Method of Growth Determination}

Three methods of growth determination by means of recurrent surveys may be distinguished, as follows:

(a) Temporary plots are used at each remeasurement and no attempt is made to remeasure identical plots. The net growth figures are obtained by subtracting the average initial volume from the average final volume for particular areas or types.

(b) Permanent plots are established initially and subsequent remeasurement is made on identical plots. The growth figures are obtained as in the first method.

(c) Permanent plots are established and remeasured as in (b) but the net growth figure is obtained by subtracting initial from final volumes for each plot and averaging individual plot increments to obtain the average for a particular area.

The first of these methods should never be used if reliable figures for growth are required; the second method is an improvement, but the last method, whereby growth is determined on an individual plot basis, is by far the best.

Examples will serve to demonstrate the basis for these conclusions.

\section{Example 1}

Table 1 tabulates data similar to that obtained by measuring 10 temporary plots in a sample area and remeasuring the area with another set at a 5-year interval. Note that the second value tabulated is not the remeasured value of the first. All measurements are independent.

Table 1 shows the calculations required to find the average yields in 1940 and 1945 and the average increment along with their standard errors. The standard error is an index of the reliability of an average and means that the true average of the area from which the sample was drawn has 67 chances out of 100 of falling within the range indicated by the standard error. Thus for the 1940 measurements the true average yield per acre of 
the area is probably $3,879 \pm 245$ cubic feet or from 3,624 to 4.124 feet, or as expressed as a percentage it should be within 6.3 per cent of 3,879 cubic feet.

Table 1

Calculation of Growth by Two Sets of Temporary Sample Plots Independently Established in the Same Area at a 5-year Interval

Volume in Hundreds of Cubic Feet per Acre
Increment

Total $\ldots \ldots \ldots \ldots \ldots \ldots \ldots \ldots \ldots \ldots, \quad 387.9$

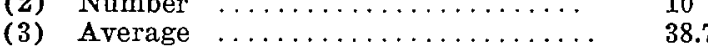

$1940 \quad 1945$

$33.5 \quad 50.5$

$1940-45$

4) Sum of squares

(5) $(\text { Total })^{2} /$ number $\ldots \ldots \ldots \ldots \ldots 15,046.6$

(6) Sum of squares of deviations ..... 539.35

(7) Standard deviation

$=\sqrt{\sqrt{(6) / n-1} \ldots \ldots \ldots \ldots \ldots \ldots \ldots} \quad \begin{aligned} & 7.74 \\ & \text { Standard error }=\ldots \ldots \ldots \ldots \ldots \ldots\end{aligned}$

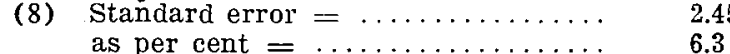

$$
\begin{aligned}
& 50.5 \\
& 41.8
\end{aligned}
$$

50.2

45.2

34.8

43.1

32.2

$\begin{array}{ll}47.1 & 39.9 \\ 38.3 & 51.8\end{array}$

$\begin{array}{ll}47.1 & 39.9 \\ 38.3 & 51.8\end{array}$

$\quad 38.9$

428.

10
42.8

42.84
$18,754.52$

4.05

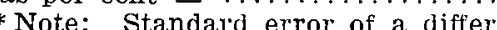
measurements

$$
\begin{aligned}
& =\sqrt{{\mathrm{S} . \mathrm{E} .2_{1}+\mathrm{S} . \mathrm{E} \cdot 2_{2}}^{2}} \\
& =\sqrt{2.45^{2}+2.11^{2}}=3.23
\end{aligned}
$$

The average growth of 405 cubic feet with which one is primarily concerned is extremely unreliable since it has a standard error of 323 cubic feet or 79.7 per cent; hence the true growth for the area might be anywhere from 82 to 728 cubic feet for a 5 -year period. Obviously this figure is almost useless for precise work, and further deductions or attempted correlations from it are merely wishful thinking.

The reason the average increment so determined is so unreliable lies in the fact that within a given year's measurements the differences in yields are so great as to obscure differences in yield between the two sets of measurements. This difficulty can be overcome either by remeasuring the same plots each time, or by greatly increasing the number of plots at each remeasurement. Example 2 explains this.

\section{Example 2}

The data used in Table 1 were actually from two sets of measurements on permanent plots when unsorted on the basis of individual plots. When the data are sorted out on the basis of identical plots it will appear as in 
Table 2 and it is possible to calculate the average increment and its standard error from individual plots.

Table 2

Calculation of Growth by Permanent Plots Remeasured at a 5-year Interval

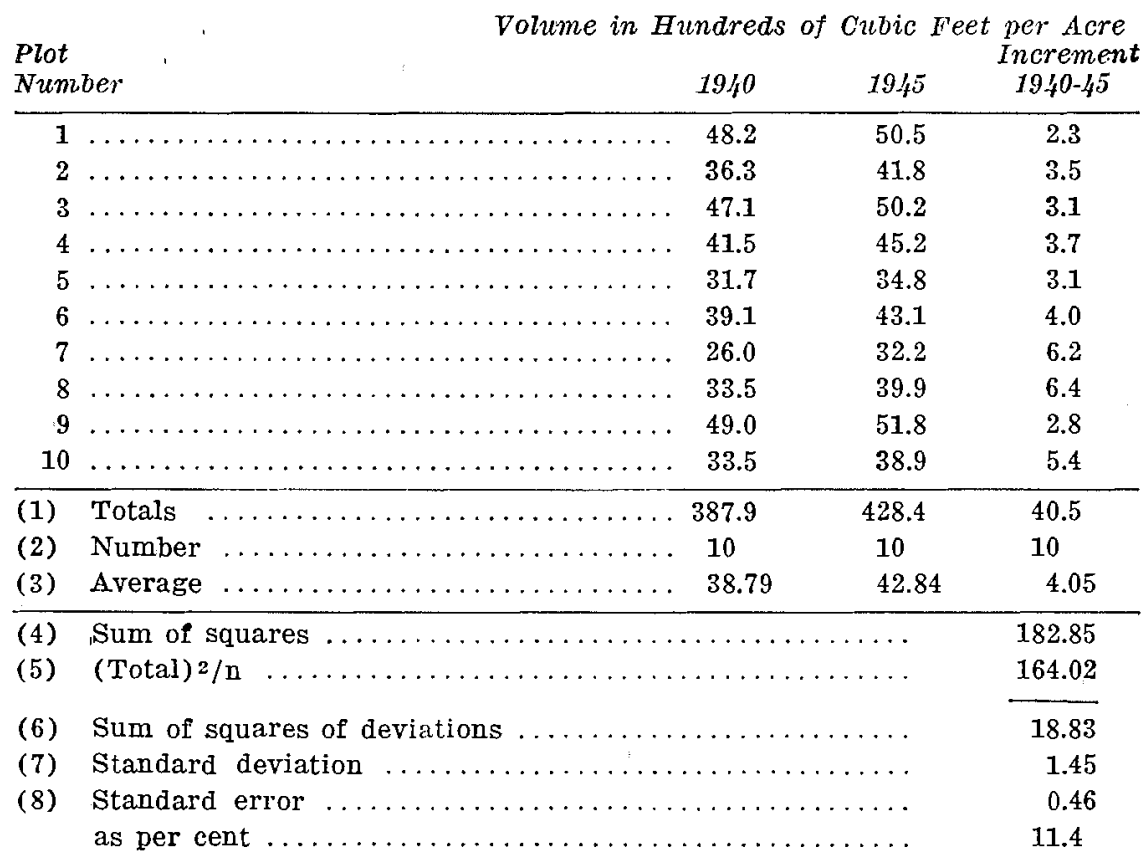

The average increment figure of 405 cubic feet obtained by paired measurements and shown in Table 2 is roughly seven times as reliable as the average from unpaired plots (Table 1), i.e., the standard errors are in the proportion of about 1 to 7 , or 11.4 per cent to 79.7 per cent. Since the same numerical data were used for purposes of example in both tables, the averages are of course identical in numerical value. In actual practice they could be expected to diverge to the extent of their standard errors. It is apparent that measur. ing identical plots at each remeasurement reduces the error in the increment enormously.

However, as mentioned under Example 1, the large error in taking independent sets of unpaired plots can be reduced by increasing the number of plots. Usually the number of independent plots required to equal the accuracy obtainable in increment on the paired plots is so great as to make such an alternative quite impractical. To illustrate, the number of plots required to reduce the standard error of Example 1 (independent plots) to that equal. To the standard error of Example 2 (paired plots) may be computed as follows: 
Required standard error of increments $=0.46$ (Table 2)

Standard error of two yields in example 1 must be reduced so that

$$
\begin{aligned}
\sqrt{\text { S.E. } 2_{1}+\text { S.E. } 2_{2}} & =0.46 \\
\text { or squaring } & \\
\text { S.E. } 2_{1}+\text { S.E. } 2_{2} & =0.2116
\end{aligned}
$$

but since S.E..$_{1}$ and S.E..$_{2}$ are practically equal then to a reasonable approximation

$$
\begin{aligned}
& \text { S.E. } 2_{1}=\frac{0.2116}{2}=0.1058 \\
& \text { S.E. }{ }_{1}=\sqrt{0.1058}=0.325
\end{aligned}
$$

Since for 10 plots the observed standard error in the yield was 2.45 , the number of plots required to obtain a standard error of 0.325 , using the theorem that the standard error varies inversely with the square root of the number of measurements, will therefore be

Number $=\left(\frac{\text { known standard deviation }}{\text { required standard error }}\right)^{2}=\left(\frac{2.45 \sqrt{10}}{0.325}\right)^{2}=569$ plots

Thus for this particular example it would have been theoretically necessary to establish and measure over 500 temporary plots at each remeasurement in order to equal the accuracy in increment obtained by the remeasurement of 10 paired plots.

When permanent plots have been established with subsequent remeasure. ment made on the same plots, and the growth has been obtained by subtracting the two average yields, it is incorrect to calculate the standard error of the difference in yields (increment) by the method in Table 1 since the two sets of data are not independent. If the standard error were calculated in such a manner it would be nuch too high, since the assumption would be that the two sets of data were quite independent of each other.

In summarizing the following points should be noted:

(a) If temporary plots are used the increment value obtained will be subject to wide errors because of the fluctuations within the average yield figures which cannot be isolated from fluctuations in growth.

(b) When permanent plots are used and the average increment is obtained by averaging the increments of individual plots the increment value will be many times more reliable than that from temporary plots, since yield fluctuations will be minimized by the pairing of the plots.

(c) When the average increment from a series of permanent plots is found by subtracting the average yields it is impossible to calculate correctly the reliability of the average so found, although the average will have the same numerical value as that obtained by averaging individual increments.

(d) The most suitable method is therefore to use permanent plots with individually calculated increments. 


\section{ERror INVOLVEd by INCORRECTLy AsSuming that Remeasurement Was Made on Identical Plots}

Serious errors in increment may be encountered even when permanent plots are used. A major source of such error is an incorrect plot boundary, i.e., the remeasurement does not measure the trees on exactly the same area as did the initial survey, either because some of the corner posts have become obliterated or because perhaps only one of four corners was permanently marked originally.

The magnitude of the errors from this source is often not fully appreciated. Example 3 illustrates this point.

Example 3

The area of plot 1 in Table 2 was 0.1 acre; the initial and final volumes per plot were 482 and 505 cubic feet; the average diameter of the stand was $10^{\prime \prime}$ D.B.H. Assume that a $10^{\prime \prime}$ tree which was included in the initial measurement was accidentally excluded at the remeasurement because of poorly defined boundaries. Table 3 shows the effect of this error.

Table 3

Typical Errors Caused by Poorly Defined Plot Boundaries

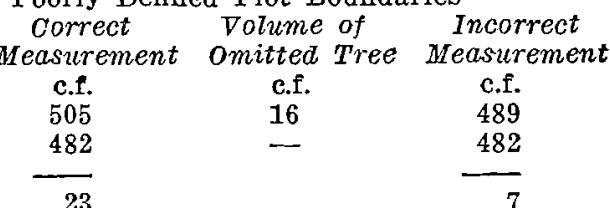

1940 yield $\ldots \ldots \ldots \ldots \ldots \ldots \ldots \ldots \ldots \ldots \ldots \ldots \ldots \ldots \ldots \ldots \ldots$
Increment $\ldots \ldots \ldots \ldots \ldots \ldots$

Error $=16$ cubic feet increment per plot in 5 years

$=160$ cubic feet per acre in 5 years

$=0.32$ cords per acre per year

$=\quad 3.3$ per cent of the initial yield

An error of a single $10^{\prime \prime}$ tree can completely distort the increment and cause an error of one-third of a cord per acre per year in the growth. For many stands this error is greater than the usual growth. Taken over a large number of plots such errors may (but not necessarily) be compensating, with the result that the average is changed but little; however, such an average would be much more unreliable than that from accurately delineated plots since the deviations from the average would be much larger, thus increasing the standard error.

The most practical way of reducing errors from this source in the case of rectangular plots is to make sure that all four corners are definitely and permanently marked. Wooden posts are not very satisfactory when the remeasurement period is 10 years or more, since under many conditions they rot away, particularly if they are of small dimensions; in addition their manufacture in the field is time-consuming. The Dominion Forest Service, this year, is using one large wooden post $\left(4^{\prime \prime} \times 4^{\prime \prime}\right.$ or larger) for one corner, and $18^{\prime \prime}$ pins of discarded No. 9 galvanized iron telephone wire with small aluminum flags for the other three corners. The advantages are: the plot itself can be located from the large post and the corners from the heavy wire 
pins: the pins are practically indestructible, cheap, and light to carry, less time is required for establishment than if wooden posts have to be cut for each corner. Experiments are also being made with 18" lengths of alloyed angled aluminum which weigh only $1 / 3$ of a pound each.

Another way to reduce errors is to use larger plots. If the plot used in the example had been one-fifth instead of one-tenth acre the error on a per acre basis would have been halved. The error in the annual increment would also be halved if the remeasurement period had been 10 instead of 5 years. Increment based on a short remeasurement period is not as reliable as that based on a longer period.

In all cases, remeasurement should be done on the trees on exactly the same area as used originally even though the original plot may have had a slight error in area.

\section{ERror from Mistakes in TALlying}

No statistical device or measurement technique can compensate for sloppy field work. When line-plots are put in for growth studies it is absolutely essential that the field men be made to realize that accurate tallies are neces. sary in estimating growth by periodic remeasurement. The growth figure is an extremely delicate one, and work which is sufficiently accurate for ordinary cruising is not good enough for measurements of growth.

It is preferable to take the previous records into the field when the reineasurement is made so that gross errors can be detected and rectified on the spot.

The effect on increment of errors in tallying will be similar to that noted in example 3.

\section{Miscellaneous Sources of Error}

Care should be taken to see that the same standard volume tables are used for each remeasurement. For the measurement of the increment it is better to use an incorrect table for all sets of data rather than, say, a more accurate table for only one set and different tables for the other. Differences in the volume tables are directly reflected in the increment.

Particular care is required in the application of height-diameter curves. The measurements for each curve must be taken from the same area at each measurement and applied to the same group of plots. It is obviously incorrect, for example, to use for the initial measurements a generalized heightdiameter curve for the whole area, and then at remeasuremnt to make up separate height-diameter curves for each site type. In such a case it may be preferable to recompile the original measurements using the curves taken from the second remeasurement if it is assumed likely that differences in curves for various sites will be greater than the difference caused by 5 or 10 years' growth.

In general it is essential that each set of measurements be compiled in exactly the same way as was done previously. Unfortunately no experimental data are readily available to show the magnitude of possible errors from the various sources. However, values taken at random from a book of volume 
tables show that an error of 5 units in form-class may give approximately 8 per cent error in volume, and 10 foot error in height from 10 to 20 per cent error in volume. Thus if, say, the yields from one remeasurement are in error by 10 per cent, this error will probably be greater than the real increment, and in any case the increment figures will be meaningless.

\section{Size of Plots}

Mention has been made of the influence of the size of the plot on some errors. It is impossible to name the best size of plot under all conditions since the requirements differ according to the size and homogeneity of the stands sampled. Generally speaking, larger plots are required for large sized timber or where the stand is very variable (as in most cut-over stands), than in smaller and more homogeneous stands. In any case the plot should not be less than one-tenth acre in area and for practical considerations it will rarely be greater than one-quarter of an acre. In any given line-plot survey the plots should be of a constant size. Different sized plots are fertile fields for errors in compilation, and in addition, make it impossible to arrive at any reasonable assessment of the reliability of the averages concerned.

A recent investigation by the Dominion Forest Service of the relative suitability of one-tenth and one-fifth acre plots in sampling immature mixed. wood stands indicated that the one-fifth acre plots were the most suitable. About 1.6 times as many one-tenth acre plots as one-fifth acre plots were required to give averages of equal reliability, and the stem number distributions on the larger plots were much less erratic. It was estimated that the output in number of plots established per crew per day would be reduced by not more than 20 per cent if one-fifth instead of one-tenth acre plots were used. For average. conditions in Eastern Canada it is believed that one-fifth acre plots are usually the most suitable size for general line-plot surveys.

\section{Number of Plots}

The number of plots which should be established in an area will depend upon the accuracy desired and upon the variability which exists on the area sampled. The accuracy of the average obtained from samples of a given area varies as the square root of the number of samples. Thus, 64 samples will give an average twice as reliable as will 16 samples.

Results from a number of surveys seem to indicate that generally from 15 to 20 samples are required to give an average with a standard error below 10 per cent, and that 3 per cent is about the lowest practical value obtainable, requiring about 300 samples.

A rule of thumb is to select whatever spacing is required to get about 20 plots in the smaller type or subdivision for which a reliable average is required.

\section{General Remarks}

The advantages of dealing with individual plot values for yield and increment have been pointed out a number of times. In order to do this, the volume of each plot must be calculated separately. While superficially 
this may appear to involve considerable additional compilation, it will be found that the amount of summarizing is greatly reduced and that rearrangements of the data may be made very simply without laborious recompilation. Compilation by individual plots is greatly facilitated if volumes are computed on the field sheets.

The initial compilation of line plot data should be very carefully, planned, with particular attention being given to the volume tables and height. diameter curves used, so that future compilations of remeasurement datia can be made upon the same basis.

\section{SUMMary}

The main points brought out in a discussion of technique for recurrent line-plot surveys for the study of growth were:

(a) Permanent rather than temporary plots should be established.

(b) Increment should be computed on an individual plot basis instead of by taking the difference in average yield between one measurement and the next.

(c) Large errors in increment may occur unless all four corners of permanent plots are definitely and permanently marked on the ground.

(d) Much greater care is required in tallying plots for growth studies than for inventory purposes.

(e) All compilations of remeasurement data must use the same methods, volume tables and comparable height-diameter curves as the original measurement. Otherwise increment figures are meaningless.

(f) Size of plots used should be based on size and variability of timber sampled. Under many conditions one-fifth acre plots appear most suitable.

(g) Number of plots used depends on required accuracy and variability of stands sampled. Not less than 20 plots should be used for a given average if a standard error of less than 10 per cent is required.

(h) Compilation of volume, etc., should be done on an individual plot basis since much summarizing is obviated and the data are more easily rearranged. 